\section{A) Check for updates}

Cite this: Nanoscale, 2020, 12, 15677

\title{
First-time synthesis of a magnetoelectric core-shell composite via conventional solid-state reaction $\uparrow$
}

\author{
Leonard F. Henrichs, (D) *a,b Xiaoke Mu, ${ }^{a}$ Torsten Scherer, ${ }^{a, c}$ Uta Gerhards, ID d \\ Stefan Schuppler, e Peter Nagel, ${ }^{e}$ Michael Merz, iD e Christian Kübel, (iD) a,c,h \\ Mohammed H. Fawey, ${ }^{a, f}$ Thomas C. Hansen (D) $g$ and Horst Hahn (D) a,h
}

\begin{abstract}
In recent years, multiferroics and magnetoelectrics have demonstrated their potential for a variety of applications. However, no magnetoelectric material has been translated to a real application yet. Here, we report for the first time that a magnetoelectric core-shell ceramic, is synthesized via a conventional solid-state reaction, where core-shell grains form during a single sintering step. The core consists of ferrimagnetic $\mathrm{CoFe}_{2} \mathrm{O}_{4}$, which is surrounded by a ferroelectric shell consisting of $\left(\mathrm{BiFeO}_{3}\right)_{x}-\left(\mathrm{Bi}_{1 / 2} \mathrm{~K}_{1 / 2} \mathrm{TiO}_{3}\right)_{1-x}$. We establish the core-shell nature of these grains by transmission-electron microscopy (TEM) and find an epitaxial crystallographic relation between core and shell, with a lattice mismatch of $6 \pm 0.7 \%$. The coreshell grains exhibit exceptional magnetoelectric coupling effects that we attribute to the epitaxial connection between the magnetic and ferroelectric phase, which also leads to magnetic exchange coupling as demonstrated by neutron diffraction. Apparently, ferrimagnetic $\mathrm{CoFe}_{2} \mathrm{O}_{4}$ cores undergo a non-centrosymmetric distortion of the crystal structure upon epitaxial strain from the shell, which leads to simultaneous ferrimagnetism and piezoelectricity. We conclude that in situ core-shell ceramics offer a number of advantages over other magnetoelectric composites, such as lower leakage current, higher density and absence of substrate clamping effects. At the same time, the material is predestined for application, since its preparation is cost-effective and only requires a single sintering step. This discovery adds a promising new perspective for the application of magnetoelectric materials.
\end{abstract}

\begin{abstract}
Received 27th March 2020, Accepted 22nd June 2020 DOI: $10.1039 / d 0 n r 02475 a$

rsc.li/nanoscale
\end{abstract}

\section{Introduction}

Core-shell type materials are of great interest in many research areas such as electronics, catalysis or biomedical applications and research activity in this field has increased steadily over the

${ }^{a}$ Karlsruhe Institute of Technology, Institute of Nanotechnology, Hermann-vonHelmholtz-Platz 1, 76344 Eggenstein-Leopoldshafen, Germany.

E-mail: leonard.henrichs@kit.edu

${ }^{b}$ Karlsruhe Institute of Technology, Institute of Applied Geosciences, Adenauerring. 20b, 76131 Karlsruhe, Germany

${ }^{c}$ Karlsruhe Institute of Technology, Karlsruhe Nano Micro Facility, Hermann-vonHelmholtz-Platz 1, 76344 Eggenstein-Leopoldshafen, Germany

${ }^{d}$ Karlsruhe Institute of Technology, Institute for Micro Process Engineering, Hermann-von-Helmholtz-Platz 1, 76344 Eggenstein-Leopoldshafen, Germany ${ }^{e}$ Karlsruhe Institute of Technology, Institute for Quantum Materials and Technologies, 76021 Karlsruhe, Germany

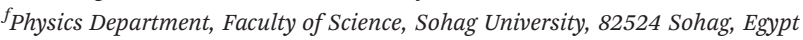
${ }^{g}$ Institut Laue-Langevin, 71 avenue des Martyrs, 38000 Grenoble, France

${ }^{h}$ Joint Research Laboratory Nanomaterials, Technische Universitaet Darmstadt, Petersenstr. 32, 64287 Darmstadt, Germany

$\dagger$ Electronic supplementary information (ESI) available. See DOI: 10.1039/ d0nr02475a past years. ${ }^{1,2}$ These materials consist of particles or grains with a central core surrounded by a shell, where core and shell have different chemical composition but usually very similar crystal structure and epitaxial crystallographic relation. ${ }^{3,4}$ However, so far only a few core-shell type materials are commercially relevant such as dielectrics for multilayer ceramic capacitors with high thermal stability fulfilling the X7R specification. ${ }^{2,3,5,6}$ In these $\mathrm{BaTiO}_{3}$-based materials, the core-shell structure leads to high thermal stability of the dielectric permittivity. More recently, they have attracted new attention in lead-free piezoelectrics, since they exhibit exceptionally high electromechanical strain, due to their core-shell microstructure. ${ }^{7,8}$

An important characteristic of these ceramics is the fact that they are produced in a one-step solid-state sintering process, where the core-shell grains are formed "in situ" due to impeded diffusion resulting in kinetically stabilized coreshell grains. ${ }^{3,4,6}$ The one-step fabrication process makes these materials interesting for applications, due to low production costs. Therefore, we want to suggest the term in situ core-shell (ISCS) ceramics for these materials, to distinguish them from nanoparticle based core-shell materials. The latter represent 
the majority of core-shell materials in current research, and consist of isolated nanoparticles which are usually produced by a multistep layer-by-layer synthesis approach such as (co) precipitation $^{9,10}$ or microemulsion. ${ }^{11}$ Such core-shell particles might be pressed and sintered to form a bulk material in a consecutive step. However, this approach is relatively elaborate and not suitable for economical synthesis of large amounts of material. Furthermore, there are a number of important drawbacks for such materials: (i) the metastable core-shell structure present in the base-particles might be lost during sintering of the bulk material due to diffusion and grain growth. (ii) To avoid losing the core-shell structure, relatively low sintering temperatures are employed, which might result in low density and thus lack of good ferroelectric properties.

Multiferroic and magnetoelectric (ME) materials are among the top research topics in materials science in the field of smart materials according to Thompson Reuters, due to their rich and complex physics and potential for applications in sensors and memory. ${ }^{12}$ Although there have been notable improvements in recent years such as for Aurivillius phases or $\mathrm{PbZr}_{0.53} \mathrm{Ti}_{0.47} \mathrm{O}_{3}-$ $\mathrm{PbFe}_{0.5} \mathrm{Ta}_{0.5} \mathrm{O}_{3}$ solid solutions, single-phase multiferroics so far exhibit ME effects that are unacceptably weak in terms of applications. $^{13,14}$ Composite magnetoelectrics on the other hand are more promising, since they offer much stronger ME coupling coefficients at ambient conditions. Many composite ME materials consist of a ferroelectric perovskite and a ferrimagnetic spinel. Due to similarities in their crystal chemistry and lattice constants, these materials possess the ability to form heteroepitaxial composites. Common ferroelectric perovskites have a cell parameter of $\sim 4 \AA$, which is within a 5\% mismatch to the main building block of the $\mathrm{CoFe}_{2} \mathrm{O}_{4}$ (CFO) structure, corresponding to half of its cell parameter $(\sim 4.19 \AA),{ }^{15}$ meaning that the distances between e.g. (100) c planes for perovskites and $(200)_{c}$ planes for spinells are similar. Many ME composite materials have been realized for example in the systems $\mathrm{BaTiO}_{3}-\mathrm{CoFe}_{2} \mathrm{O}_{4},{ }^{15-17}$ or $\mathrm{BiFeO}_{3}-\mathrm{CoFe}_{2} \mathrm{O}_{4},{ }^{18,19}$ and some also with core-shell type structures such as core-shell fibers. ${ }^{20,21}$

\section{Magnetoelectric composites: challenges and opportunities}

The most common connectivity schemes of ME composites are $0-3,2-2$ and $1-3$, corresponding to particles dispersed in a matrix, layered films and parallel fibers embedded in a matrix, respectively. Each type of composites has specific drawbacks. For example, 0-3 composites prepared from core-shell or conventional nanoparticles often exhibit weak coupling due to their polycrystalline nature, while 2-2 composites display low ME coupling due to substrate clamping. For 1-3 composites, high leakage currents have been reported due to percolating CFO pillars with relatively low resistivity. ${ }^{22,23}$ On the other hand, no ME in situ coreshell ceramic has been reported so far, although they have the potential to circumvent some of the aforementioned drawbacks, all the while being predestined for applications due to their wellestablished, cost-effective production process.
Here, we report for the first time that a magnetoelectric (ME) core-shell ceramic has been synthesized in a conventional solidstate synthesis process. The previously reported multiferroic clusters $^{24}$ which exhibit exceptional ME coupling, are identified as core-shell grains with a magnetic core consisting of CFO, which is surrounded by a ferroelectric perovskite-type matrix.

The in situ ME core-shell ceramics combine characteristics from different composite classes, namely 0-3 connectivity (usually polycrystalline bulk ceramics) and epitaxial intergrowth, which usually occur in 2-2 and 1-3 thin-film type composites (see Fig. 1). The new 0-3 epitaxial composite has the potential to eliminate drawbacks of other composite classes. This adds an exciting new perspective for ME composites.

\section{Sample synthesis and description}

All experimental results presented in this work were obtained on polycrystalline ceramics with the overall composition $\left(\mathrm{BiFe}_{0.9} \mathrm{Co}_{0.1} \mathrm{O}_{3}\right)_{0.4}-\left(\mathrm{Bi}_{1 / 2} \mathrm{~K}_{1 / 2} \mathrm{TiO}_{3}\right)_{0.6}$ (BFC-BKT) except where explicitly stated. Their crystal structure as well as ferroelectric and magnetic properties have been reported elsewhere. ${ }^{24}$ For more information on sample appearance, microstructure and crystal structure see ESI (Fig. S1†).

Samples were prepared via conventional solid-state synthesis from $\mathrm{Bi}_{2} \mathrm{O}_{3}, \mathrm{Fe}_{2} \mathrm{O}_{3}, \mathrm{TiO}_{2}$, $\mathrm{CoO}$ (all Sigma Aldrich), and $\mathrm{K}_{2} \mathrm{CO}_{3}$ (Alfa-Aesar) (all 99.9\% purity) powders as starting materials, which were mixed according to desired stoichiometry. Milling was done in a Dynomill Typ KDLA by Willy A Bachofen with yttria-stabilised zirconia (YSZ) (Tosoh, Japan) beads (2 mm) in propan-2-ol for 1 hour to ensure homogeneity. Mixed powder was calcined at $800{ }^{\circ} \mathrm{C}$ for $4 \mathrm{~h}$ in air. The resultant product was ground using pistil and mortar and sieved through a $300 \mu \mathrm{m}$ mesh. 1 wt\% Glascol HA-40 binder (Allied Colloids, UK) and propan-2-ol was added to the powder, which was mixed again using the same procedure as before. The resultant slurry was dried via distillation and the final powder was pressed into pellets of $8 \mathrm{~mm}$ diameter and 3-5 $\mathrm{mm}$ thickness in two-step process: (i) uniaxial pressing at $50 \mathrm{MPa}$ and (ii) cold isostatic pressing at $300 \mathrm{MPa}$ for 15 minutes. These pellets were sintered in a bed of calcined powder of the same composition in a covered alumina crucible at $1065^{\circ} \mathrm{C}$ for $2 \mathrm{~h}$. During heating, the binder was burnt out slowly with a ramp rate of $50{ }^{\circ} \mathrm{C} \mathrm{h}^{-1}$ up to $600{ }^{\circ} \mathrm{C}$ followed by a fast rate of $300{ }^{\circ} \mathrm{C} \mathrm{h}^{-1}$. The pellets were cooled to $600{ }^{\circ} \mathrm{C}$ at $150{ }^{\circ} \mathrm{C} \mathrm{h}^{-1}$ so that thermal shock was reduced. The conditions for synthesizing $\left(\mathrm{BiFeO}_{3}\right)_{0.4^{-}}$ $\left(\mathrm{Bi}_{1 / 2} \mathrm{~K}_{1 / 2} \mathrm{TiO}_{3}\right)_{0.6}$ ceramics for neutron diffraction experiments were the same. For detailed synthesis see ref. 25 .

\section{Experimental results}

In order to gain a better understanding of the microstructure and chemical composition of the grains we had previously referred to as multiferroic clusters, a thin lamella was investigated by TEM. Those results are displayed in Fig. 2 . 

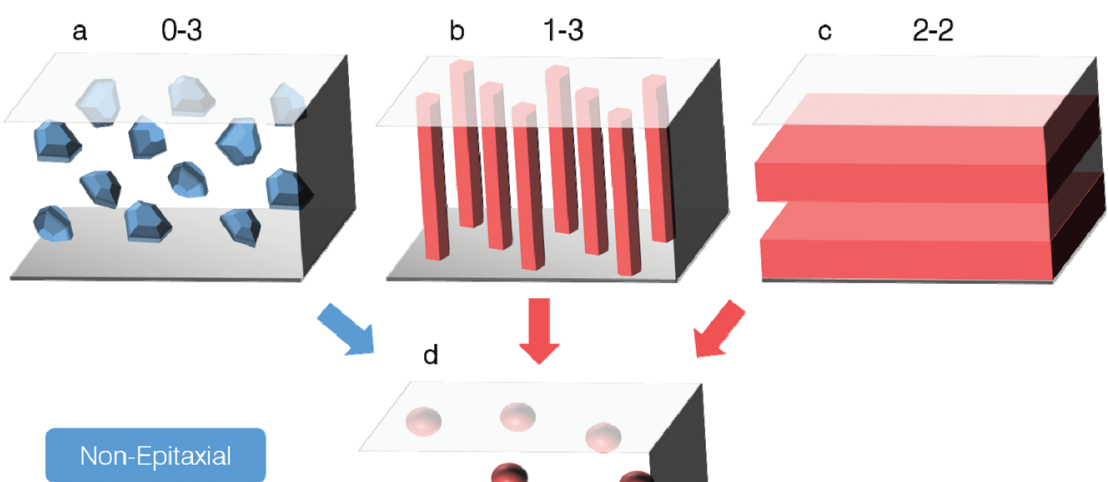

Epitaxial

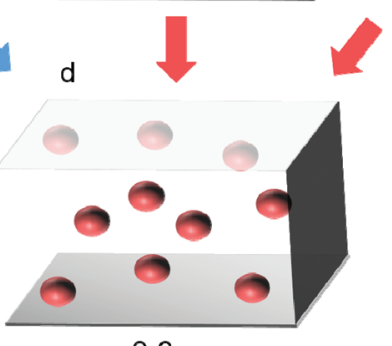

$0-3$

Fig. 1 Schematics of magnetoelectric composites with different connectivity schemes and crystallographic relation between constituents. (a) Composite with 0-3 connectivity and no epitaxial relation between grains and matrix (polycrystalline solid). (b, c) Heteroepitaxial composites with 1-3 and 2-2 connectivity respectively and epitaxial intergrowth between constituents. (d) The core-shell composite as reported here, combines $0-3$ connectivity that is usually realized in solely polycrystalline materials with epitaxial intergrowth as known from thin-film type $1-3$ or 2-2 heteroepitaxial composites.
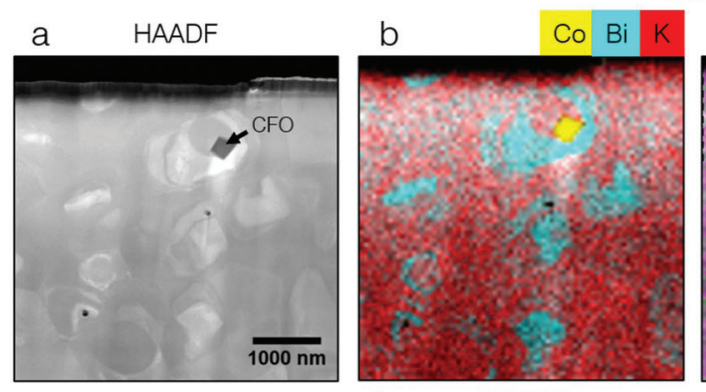

EDX
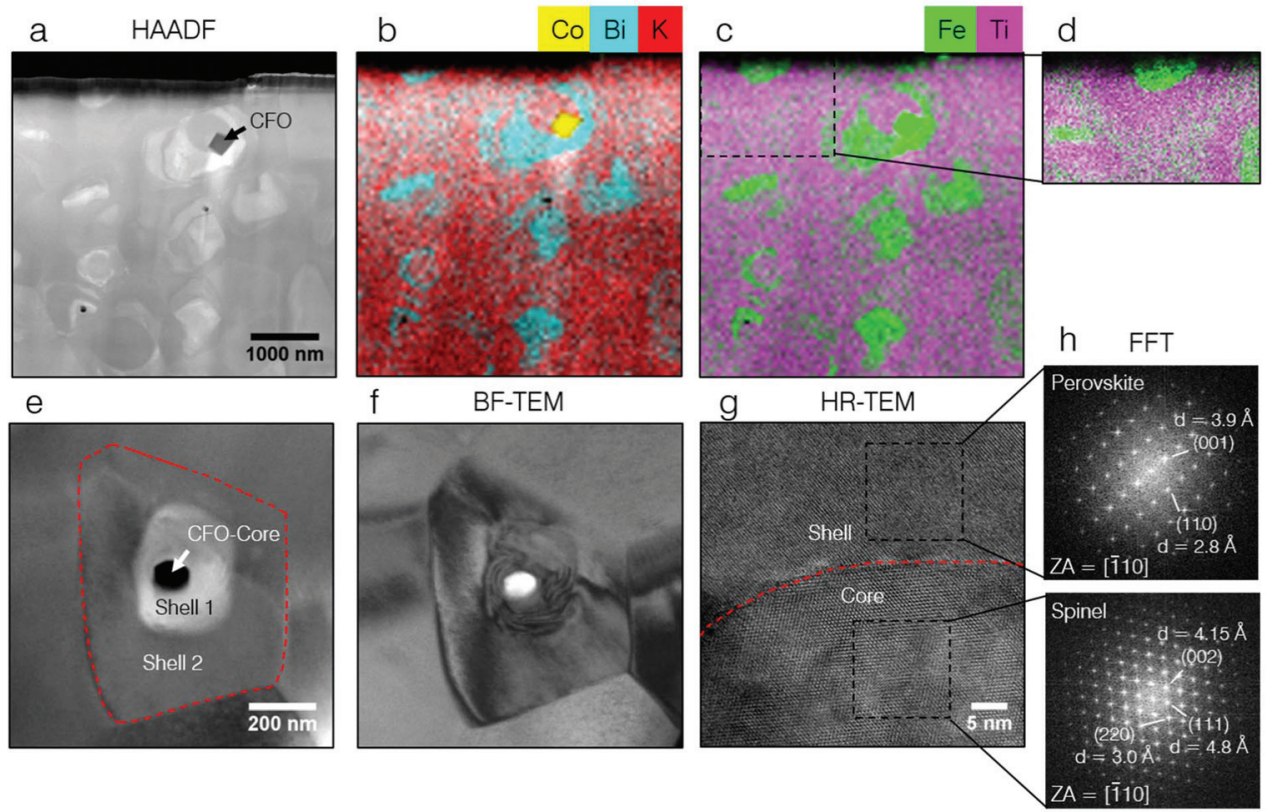

Fig. 2 TEM characterization of BFC-BKT ceramics. ( $a, b, c)$ High-angle annular dark-field (HAADF)-STEM image and corresponding EDX elemental composition maps. (b) Elemental distribution of $\mathrm{Co}$, Bi and K. (c, d) Elemental distribution of Fe and Ti. From the EDX-maps it becomes obvious that $\mathrm{BiFeO}_{3}$-rich regions exist, within a $\mathrm{Bi}_{1 / 2} \mathrm{~K}_{1 / 2} \mathrm{TiO}_{3}$-rich matrix. The $z$-sensitive $\mathrm{HAADF}$ is dominated by the $\mathrm{Bi}$ content. A CoFe $\mathrm{O}_{4}$ grain can be easily distinguished from the matrix by its Co content and low intensity in HAADF (see (a) and (b)). (e, f) HAADF and bright-field (BF) TEM images of a coreshell grain respectively. Grain boundaries are emphasized by red dashed lines. (e) Shows a typical core-shell grain where a CoFe $\mathrm{O}_{4}$ core is surrounded by a first $\mathrm{BiFeO}_{3}$-rich shell and a second $\mathrm{Bi}_{1 / 2} \mathrm{~K}_{1 / 2} \mathrm{TiO}_{3}$-rich shell. (g) High-resolution (HR)-TEM of a core-shell interface region along the [1̄î0]-zone axis. (h) Fast-Fourier-Transforms (FFTs) of the core and shell region from (g). The spinel structure shows an extra reflection (111) spinel $_{\text {(or }}$ $\left.\left(\frac{1}{2}, \frac{1}{2}, \frac{1}{2},\right)_{\text {Perovskite }}\right)$ compared to the perovskite structure, while they exhibit $6 \pm 0.7 \%$ lattice mismatch. HR-TEM proves an epitaxial relation between core and shell, since both structures are well aligned.

EDX elemental maps (Fig. $2 \mathrm{~b}$ and $\mathrm{c}$ ) reveal that the sample is chemically inhomogeneous. It is obvious that Bi is spatially correlated with $\mathrm{Fe}$ while $\mathrm{K}$ is correlated with Ti.
Thus, we observe that a $\mathrm{BiFeO}_{3}(\mathrm{BFO})$-rich phase exists inside a $\mathrm{Bi}_{1 / 2} \mathrm{~K}_{1 / 2} \mathrm{TiO}_{3}$ (BKT)-rich matrix. Both phases have a perovskite crystal structure. Furthermore, there is a third phase 
which is $\mathrm{CoFe}_{2} \mathrm{O}_{4}$ (CFO). A CFO grain can be easily identified by its Co content in Fig. 2b. All three phases are distinguishable in high-angular-annular dark-field (HAADF)-STEM, which is sensitive to the average atomic number and thus mainly reveals the differences in Bi concentration, which is by far the heaviest element in the material. The majority of CFO grains, exhibit a core-shell type structure. Fig. 2e shows an example of a core-shell type grain with a CFO-subgrain as the core, which is surrounded by an "inner" BFO-rich shell and an "outer" BKT-rich shell in an onion-type structure. The grain boundaries of the host grain and the core can be easily identified in bright-field (BF) TEM. We will see later by PFM that the BFO-rich phase is ferroelectric and the BKTrich matrix exhibits relaxor properties (see Fig. 2). Accordingly, we observe a ferroelectric domain structure in BF-TEM in the BFO-rich phase (see Fig. 2f). For the CFO cores that were investigated by TEM $(>20)$, we observe that these have always at least a common interface or are entirely surrounded by the ferroelectric BFO-rich phase. This lead to an erroneous interpretation of the core-shell structures as single-phase multiferroic grains in the past. ${ }^{24}$ However, the high spatial resolution of TEM, clearly reveals the multiphase nature of the previously termed multiferroic clusters. High-resolution (HR) TEM confirms an epitaxial relation between core and shell. The Fourier transforms (Fig. 2h) of the HR-TEM image of a CFO core and its perovskite shell, demonstrate the coherent crystal structure with good lattice alignment. As outlined in the introduction, spinells and perovskites can form epitaxial composites where half of the unit cell of CFO corresponds to the full unit cell of a perovskite. We observe a lattice mismatch of $6 \pm 0.7 \%$, according to the distances between (100) and (200) lattice planes for perovskite and CFO, respectively. This lattice mismatch relaxes by dislocations that occur at the core-shell grain boundary (see ESI Fig. S4 $\dagger$ ). As observed by HR-TEM, there is one lattice mismatch per 13 to 19 lattices (roughly 1 dislocation per 5 to $7 \mathrm{~nm}$ ) in the perovskite phase, which fits well to the mismatch of $6 \%$.

The composition of the BFO- and BKT-rich perovskite phases and of CFO was determined by electron probe microanalysis (EPMA) and STEM-EDX. The compositions given in this work were determined from EPMA except for CFO cores where the high lateral resolution of STEM-EDX was necessary. For BFO-rich phases, we find the composition $\mathrm{Bi}_{0.88 \pm 0.04} \mathrm{~K}_{0.18 \pm 0.03} \mathrm{Fe}_{0.66 \pm 0.08} \mathrm{Ti}_{0.36 \pm 0.08} \mathrm{Co}_{0.03 \pm 0.01} \mathrm{O}_{2.89 \pm 0.02}$, which corresponds to a stoichiometry of $x=0.66 \pm 0.06\left(\left(\mathrm{BiFeO}_{3}\right)_{x^{-}}\right.$ $\left.\left(\mathrm{Bi}_{1 / 2} \mathrm{~K}_{1 / 2} \mathrm{TiO}_{3}\right)_{1-x}\right)$ (Co is disregarded). The composition of the BKT-rich matrix $\mathrm{Bi}_{0.75 \pm 0.03} \mathrm{~K}_{0.3 \pm 0.02} \mathrm{Fe}_{0.37 \pm 0.05} \mathrm{Ti}_{0.65 \pm 0.04} \mathrm{Co}_{0.04 \pm 0.01}$ $\mathrm{O}_{2.9 \pm 0.01}, x=0.37 \pm 0.03$ is close to the intended overall composition of $x=0.4$. Uncertainties are given by the standard error across all measurements. For CFO the measured composition corresponds to the theoretical composition within the experimental error. It is doped only with minor amounts of $\mathrm{Ti}$ (approx. 0.2 at\%). Furthermore, grain boundaries also exhibit a higher BFO content as shown by the higher resolution EDX map in Fig. 2d.
In order to understand the microstructure-property relations in the material, we will give an outline of the materials electronic and magnetic microstructure.

The magnetic force-microscopy (MFM) image in Fig. 3b displays several CFO cores (marked by dashed circles). All of them display a core-shell type structure as evident from topography (Fig. 3c), where the magnetic cores display a characteristic round shape (marked cyan) and are situated rather centrally in their host grains (red dashed lines). Like in HAADF imaging, we can distinguish the three different phases by SEM in BSE mode, since this imaging mode is also sensitive to the average atomic number. Thus, we note that the bright regions in SEM-BSE are BFO-rich. It becomes obvious that the BFO-rich phase displays enhanced piezoelectricity by comparing the PFM and SEM-BSE images (Fig. 3e and f) whereas the BKTrich phase displays a PFM amplitude close to 0 . Note that we determined the so-called PFM background signal ${ }^{26,27}$ by recording the PFM X- and Y-Amplitude on a non-piezoelectric reference sample and a number of non-piezoelectric contaminant particles. Both values were in good agreement. The value of the PFM background signal was $0.24 \pm 0.05$ in a.u. and was set to 0 in the PFM-scale used in Fig. 3. The error is the standard deviation across all reference measurements.

Surprisingly, some of the CFO cores (marked by cyan circles in Fig. 3e) exhibit a PFM-signal that is different from the background value. This fact is highlighted in Fig. 3d, which shows line profiles through a CFO core (1) (see Fig. 3e magnified panel) and a ferroelectric BFO-rich region (2) for comparison. The PFM X-Amplitude measured across the CFO core (1) has a value of $\sim-0.13$ which is different from the PFM background signal also when considering the error. Thus, it appears to exhibit piezoelectricity. We want to consider whether these non-zero PFM-signals could be due to measurement artifacts, or arise from actual piezoactivity. We have carried out PFM experiments on non-epitaxial CFO particles present in BFCBKT ceramics, which display a 0 PFM signal homogeneously across the whole particle and interface between particle and matrix. Furthermore, PFM probes the piezoresponse very locally and we think that the lateral resolution as well as the probing depth, which should not be larger than $200 \mathrm{~nm}$ are small enough to probe only the CFO core and not a mixed signal from the CFO core and for example a ferroelectric region underneath it. We see a saturation of the PFM signal over a distance of approx. $100 \mathrm{~nm}$ around the particle's centre, which supports that the lateral resolution is good enough. Furthermore, we estimated that the amplitude of the PFMsignal of the CFO core in relation to that of the ferroelectric region as displayed in Fig. 3b (approx. 1:3) are realistic, by comparing it to a known ferreoelctric reference sample $\mathrm{LiNbO}_{3}$. The local piezoelectric coefficients of the ferroelectric regions are similar to $\mathrm{LiNbO}_{3}$ with a moderate $\mathrm{d}_{33}$ coefficient of $20 \mathrm{pm} \mathrm{V}^{-1} .^{28}$ Therefore, the amplitude to the CFO core's PFM-signal seems realistic. For a detailed discussion on possible artifacts, PFM resolution and amplitude, see ESI. $\dagger$

We propose that the epitaxial strain from the polar perovskite matrix causes a polar distortion of the spinel crystal struc- 

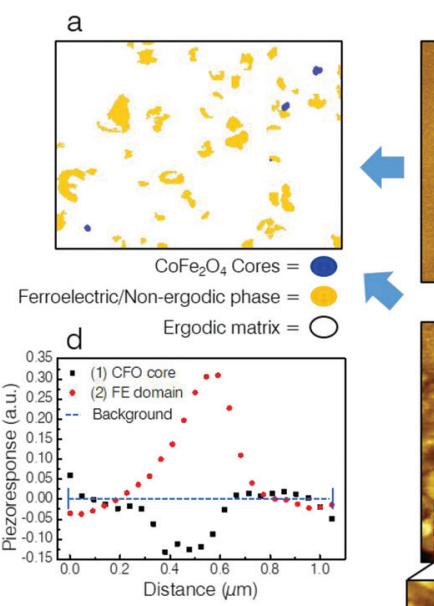

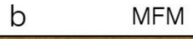

b

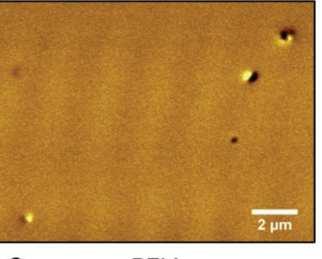

e

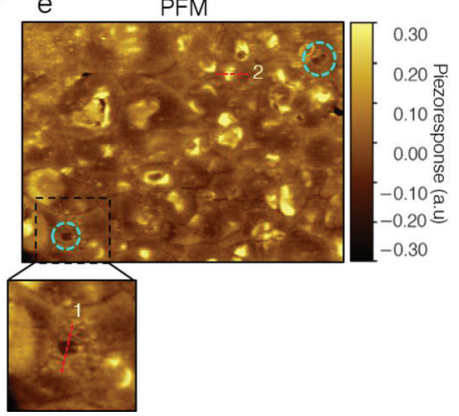

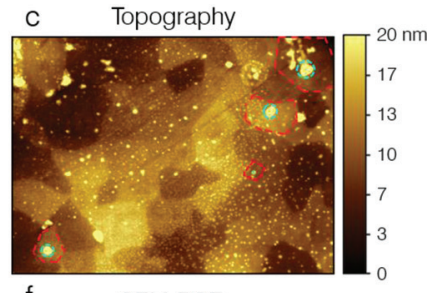

f SEM-BSE

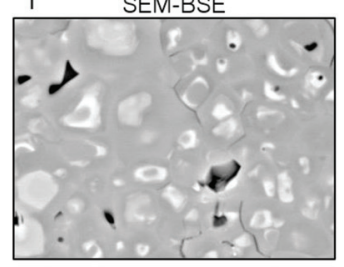

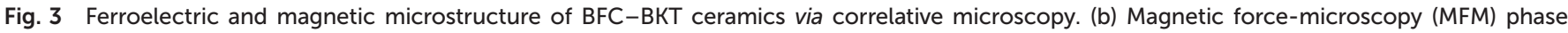

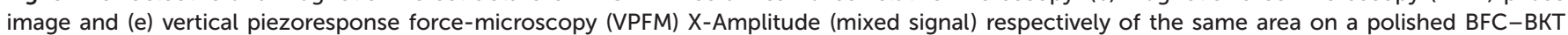

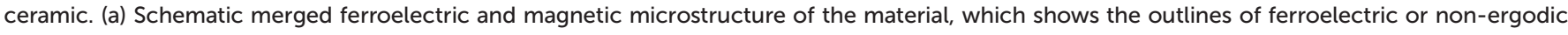

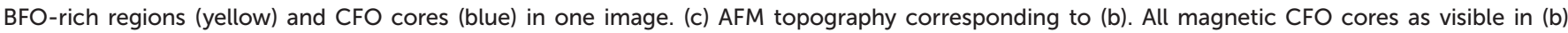

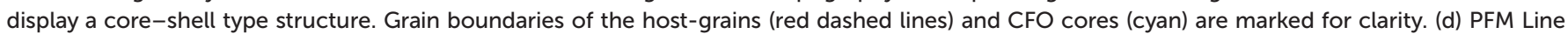

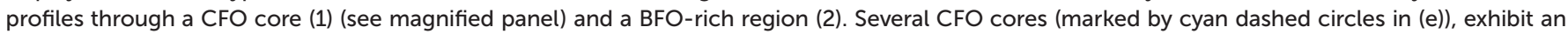

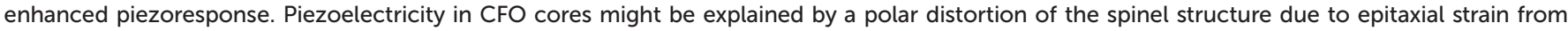

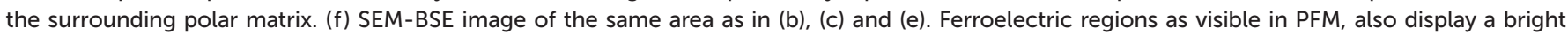

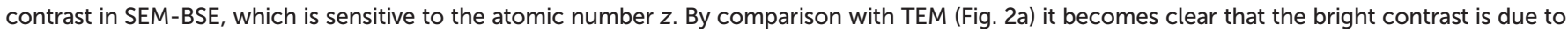

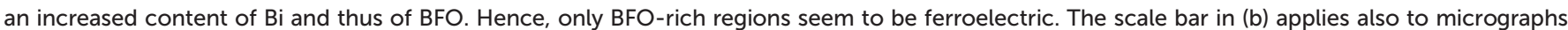
(c) (e) (f), which all show the exact same sample region.

ture, which in turn leads to piezoelectricity in CFO. The majority of cores have sizes between $50-200 \mathrm{~nm}$. It is reasonable to assume that epitaxial strain from the matrix is not fully relaxed over these length scales (for example by dislocations), and thus has a significant influence on the cores' structure and properties. Piezoelectricity of CFO might play an important role for the observed strong ME coupling. Scanning-transmission X-ray microscopy (STXM) was employed to support results by PFM, which further support strong coupling between core and shell (see ESI†).

In previous work, we demonstrated the relaxor type properties of the material. ${ }^{24}$ Thus, we identify the BFO-rich phase as non-ergodic, displaying long-range ferroelectric order, and the BKT-rich matrix as ergodic, which does not maintain longrange ferroelectric order in absence of a sufficiently large electric field. This is consistent with the literature where in the system $\left(\mathrm{BiFeO}_{3}\right)_{x}-\left(\mathrm{Bi}_{1 / 2} \mathrm{~K}_{1 / 2} \mathrm{TiO}_{3}\right)_{1-x}$, $\mathrm{BFO}$ richer compositions $(x>0.6)$ are reported to display rather classic ferroelectric properties, while BKT richer compositions $(x<0.4)$ are classified as relaxor ferroelectrics. ${ }^{25,29,30}$ Ferroelectricity in BFO-rich phases is further corroborated by selected area diffraction in TEM, which reveals the presence of superstructure reflections due to anti-phase octahedral tilting around the $[111]_{c}$ direction $\left(\mathrm{a}^{-} \mathrm{a}^{-} \mathrm{a}^{-}\right.$in glazer notation), which indicates a rhombohedrally distorted perovskite structure like in bulk BFO (see ESI Fig. $\mathrm{S} 1 \dagger) .{ }^{31}$ Ozaki et al., found the same superstructure reflection in ceramics with composition $x=0.6$ which is in line with the composition that we determined by EPMA. ${ }^{32}$ The increased BFO content at grain boundaries visible in Fig. 3d furthermore explains the enhanced piezoresponse as observed in PFM (see Fig. 3e). We note that when comparing Fig. $3 \mathrm{~b}$ and $\mathrm{f}$, it becomes clear that not all regions that have a dark contrast in SEM-BSE are also magnetic. From correlative SEM, PFM, MFM and EPMA elemental mapping we conclude that these must consist of antiferromagnetic $\alpha-\mathrm{Fe}_{2} \mathrm{O}_{3}$ since they exhibit no magnetic stray field in MFM and no increased Co content (see ESI Fig. S4 $\dagger$ ).

The content of all relevant phases in the sample was estimated using different techniques (see ESI† for details).

In the light of these new findings, previous results by neutron diffraction might now be understood. Previously, we had found an extremely high Néel-temperature $T_{\mathrm{N}}=670 \pm 10 \mathrm{~K}$ for $\mathrm{BFC}-\mathrm{BKT}$, which is even above the one reported for pure BFO $(650 \mathrm{~K})$ although a much lower $T_{\mathrm{N}}$ is expected due to the significantly decreased Fe content (i.e. BFO: 20 at\%, BFC-BKT: 7.2 at $\%) .{ }^{24}$ However, since we now know that the material contains core-shell type grains where CFO is epitaxially intergrown with a BFO-rich shell, the high $T_{\mathrm{N}}$ can be understood in terms of magnetic exchange coupling between the two phases. Magnetic transition temperatures of BCF-BKT and CFO were probed by temperature dependent neutron diffraction by monitoring the intensity of magnetic reflections that can be assigned to a specific phase in contrast to magnetometry data. Fig. 4 shows the magnetic peak height $v s$. temperature for CFO 


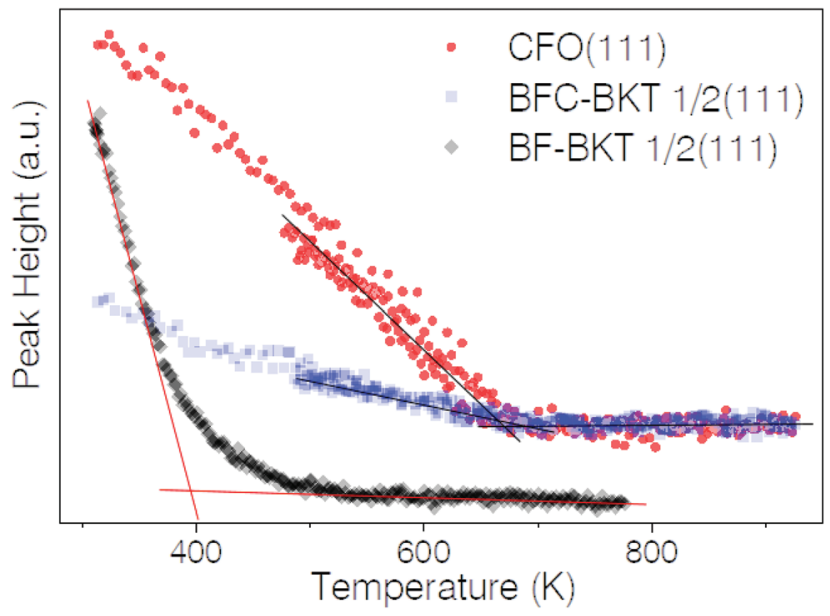

Fig. 4 Neutron magnetic peak height vs. temperature. Peak heights of antiferromagnetic $\frac{1}{2}(111)$ peak $(d=4.57 \AA)$ for $\left(\mathrm{BiFe}_{0.9} \mathrm{Co}_{0.1} \mathrm{O}_{3}\right)_{0.4}-$ $\left(\mathrm{Bi}_{1 / 2} \mathrm{~K}_{1 / 2} \mathrm{TiO}_{3}\right)_{0.6}$ (BFC-BKT, blue squares) and $\left(\mathrm{BiFeO}_{3}\right)_{0.4}-$ $\left(\mathrm{Bi}_{1 / 2} \mathrm{~K}_{1 / 2} \mathrm{TiO}_{3}\right)_{0.6}$ (BF-BKT, black diamonds) and the magnetic (111) peak ( $d=4.83 \AA$ ) for the CFO secondary phase inside BFC-BKT samples (red circles). The transition temperature for CFO and BFC-BKT are the same within experimental accuracy, while it is much lower for BF-BKT. The peak heights of $\frac{1}{2}(111) \mathrm{BF}-\mathrm{BKT}$ were normalized and offset for clarity.

Table 1 Magnetic transition temperatures from neutron diffraction vs. temperature. Errors are calculated from linear fitting of the curves and propagation of uncertainty

\begin{tabular}{|c|c|}
\hline$T_{\mathrm{N}}, T_{\mathrm{C}}$ in $\mathrm{K}$ & Phase \\
\hline $688.6 \pm 9.7$ & $\left(\mathrm{BiFe}_{0.9} \mathrm{Co}_{0.1} \mathrm{O}_{3}\right)_{0.4}-\left(\mathrm{Bi}_{1 / 2} \mathrm{~K}_{1 / 2} \mathrm{TiO}_{3}\right)_{0.6}$ \\
\hline $680.6 \pm 6.7$ & $\mathrm{CFO}$ (secondary phase in BFC-BKT samples) \\
\hline $399.1 \pm 1.4$ & $\left(\mathrm{BiFeO}_{3}\right)_{0.4}-\left(\mathrm{Bi}_{1 / 2} \mathrm{~K}_{1 / 2} \mathrm{TiO}_{3}\right)_{0.6}$ \\
\hline
\end{tabular}

(111) and the antiferromagnetic $\frac{1}{2}$ (111) peak associated to the BFO-rich phase.

The magnetic transition temperatures are determined by the intercept of the linear fits to the curve before and after the transition. The curves include points both from ramping temperature up and down, and both directions were fitted to determine transition temperatures. Results are summarized in Table 1.

The transition temperatures for BFC-BKT and CFO are identical within the experimental error. The influence of adding Co can be clearly seen for $\left(\mathrm{BiFeO}_{3}\right)_{0.4}-\left(\mathrm{Bi}_{1 / 2} \mathrm{~K}_{1 / 2} \mathrm{TiO}_{3}\right)_{0.6}$ (BF-BKT), whose $T_{\mathrm{N}}$ is approx. $280 \mathrm{~K}$ lower than for BFC-BKT. We know from EPMA and STEM-EDX measurements, that the perovskite phases are only slightly doped by Co (approx. 0.6-0.8 at\%), which should not have such drastic effect on $T_{\mathrm{N}}$. On the other hand $T_{\mathrm{N}}$ for BF-BKT is in good agreement with literature for related compounds $\left(\mathrm{BiFeO}_{3}\right)-\left(\mathrm{Bi}_{1 / 2} \mathrm{Na}_{1 / 2} \mathrm{TiO}_{3}\right)$, $\left(\mathrm{BiFeO}_{3}\right)-\left(\mathrm{BaTiO}_{3}\right)$ or $\left(\mathrm{BiFeO}_{3}\right)-\left(\mathrm{PbTiO}_{3}\right)^{33,34}$ where $T_{\mathrm{N}}$ decreases approx. linearly with decreasing Fe content and is far below that of pure BFO. Therefore, we conclude that the coinciding, high transition temperatures of BFC-BKT and CFO must be due to magnetic exchange coupling in core-shell grains, which is in agreement with our microstructural observations. Close to $T_{\mathrm{N}}$, magnetic order is maintained at least in a thin layer of the shell around the CFO core. However, upon crossing $T_{\mathrm{C}}$, CFO can no longer sustain magnetic order in the shell and both undergo magnetic transition simultaneously.

As outlined in the introduction, ME materials bear great potential for applications. One example for potential applications is a as magnetic field sensor. ${ }^{35}$ Here, the material should exhibit a high direct ME coupling effect. Fig. 5 shows a PFM under in situ magnetic field study, demonstrating strong direct ME coupling in this system. Images were recorded in

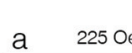

$150 \mathrm{Oe}$ $75 \mathrm{Oe}$

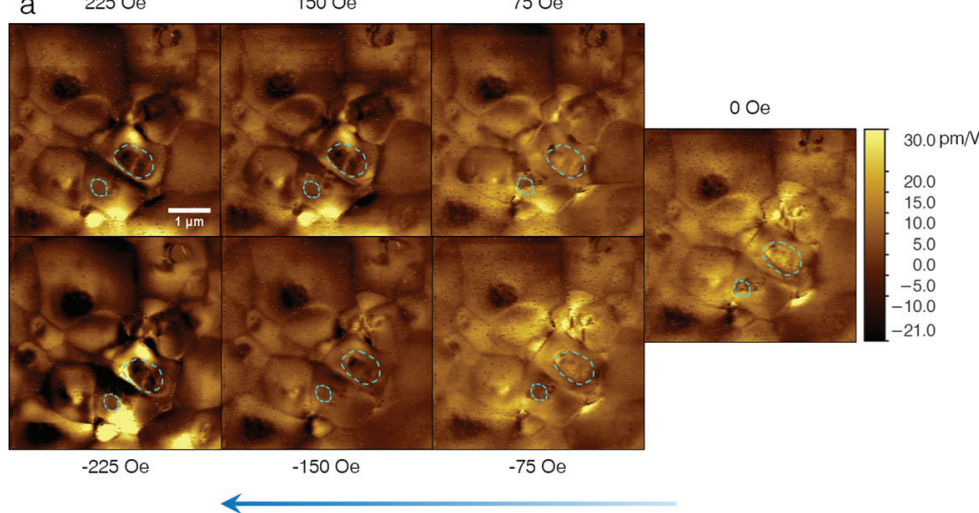

b Topography

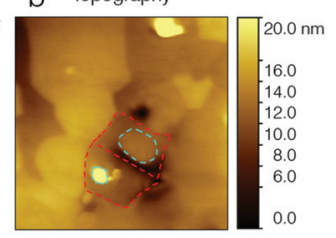

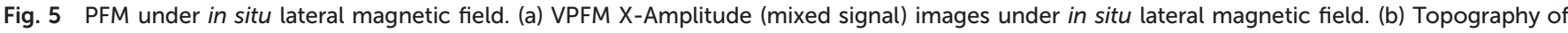

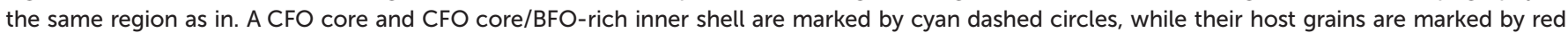

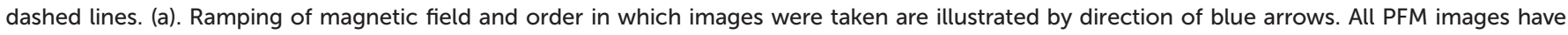

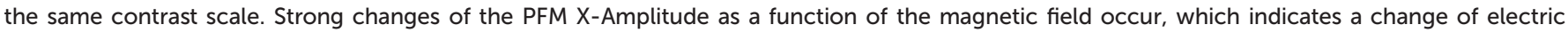
polarization as a function of magnetic field. 
the order as indicated by the blue arrows, i.e. starting from 225 Oe and ending with -225 Oe.

Changes of the domain configuration as function of inplane magnetic field are clearly observable. The effect is particularly strong for one grain with oval shape that is marked by a cyan dashed circle in Fig. 5. This oval grain is reminiscent of a core-shell grain as depicted in Fig. 2e. As in previous work, we used a ferroelectric reference sample, periodically poled lithium niobate (PPLN), to quantify the piezoresponse. ${ }^{24}$ The above measurements confirm the large ME coupling effects of this core-shell system. Additionally, we marked another CFO core that seems to be responsible for ME coupling. ME switching events occur primarily in the host grains of the two cores marked by cyan dashed circles in Fig. 5, or in the neighboring grains. In contrast, ME coupling effects were not observed in grains further away.

As found in previous work, ${ }^{24}$ we observe that changes of the domain configuration are independent of the magnetic field direction. This behavior can be explained by stress-strain mediated coupling, via magnetostriction and piezoelectricity. We certainly expect a strong stress-strain mediated coupling between core and shell, due to the epitaxial interconnection. Coupling effects to neighboring grains as observed above, are also feasible via stress-strain mediated ME coupling. PFM indicates, that some regions become ferroelectric only under magnetic field. This might be explained by the relaxor-like nature of the material. ME coupling between magnetic cores and ferroelectric inner-shells could establish long-range ferroelectric order in previously ergodic shells under magnetic field, due to a change in the mechanical and electronic boundary conditions. Therefore, the ferroelectric inner shells might act as nucleation sites for the development of long-range ferroelectric order in shells that possess relaxor properties in the absence of a sufficiently large magnetic field. This effect could lead to a larger ME effect in non-ergodic relaxor-type materials as compared to classic ferroelectrics, due to the much lower remanent polarization in relaxor ferroelectrics. In addition we have carried out macroscopic measurements of ME coupling using SQUID magnetometry under applied electric field (see ESI, Fig. S2 $\dagger$ ). These measurements suggest a macroscopic coupling coefficient of $\alpha_{\mathrm{c} \text {,eff }}=1.50 \times 10^{-11} \mathrm{~s} \mathrm{~m}^{-1}$. This coefficient is much lower than the local coupling coefficient as reported above. However, the coupling coefficient can be understood when considering (i) the low volume concentration of ME core-shell grains and (ii) the fact that the samples were not textured. A more detailed discussion is given in the ESI. $\dagger$

\section{Outlook and conclusion}

In this work, we report the first synthesis of a magnetoelectric core-shell ceramic via conventional solid-state synthesis. We describe the resulting core-shell material by using the term in situ, since core-shell grains form in situ during the (singlestep) sintering process. Therefore, we want to suggest the term in situ core-shell (ISCS) ceramics to distinguish them, from other types of core-shell materials that are usually based on pre-formed core-shell nanoparticles. ISCS ceramics combine advantages from different composite classes, namely 0-3 connectivity and epitaxial intergrowth, which usually only occur in 2-2 and 1-3 type composites with the potential to eliminate their drawbacks. Apart from the obvious practical advantage of ISCS ceramics regarding the simple production process, they should also offer several technical advantages over other ME composites. These are: (i) large ME coupling due epitaxial intergrowth of ferroelectric and magnetic phase; (ii) no substrate clamping as for 2-2 layered thin-film systems; (iii) no short-circuit problem as for 1-3 columnar composites and (iv) an improved density compared to other (nanoparticle based) 0-3 bulk systems, ${ }^{22,23}$ resulting in high resistivity and good ferroelectric properties as demonstrated before. ${ }^{24}$

We attribute the exceptional ME coupling properties of the core-shell grains to their epitaxial intergrowth leading to magnetic exchange coupling between core and shell and presumably to piezoelectricity in CFO as observed by PFM.

It is known from literature, that the number and size of core-shell grains can be tailored by choosing the right kinetic balance between crystal growth, diffusion and grain-boundary migration processes responsible for their formation. ${ }^{3,6}$ Thus, a material can be tailored with an optimized concentration of CFO cores inside a ferroelectric or relaxor matrix. However, we need to stress that the fabrication process as detailed here, does lead to impurities such as larger non-epitaxial CFO and $\mathrm{Fe}_{2} \mathrm{O}_{3}$ impurities which should be optimized before utilization in practical magnetoelectric devices. Such a material might then be processed similar to those core-shell ceramics fulfilling the X7R temperature stability criteria, which are used for multilayer capacitors. ${ }^{3,5}$ We envisage a ME core-shell ceramic that is tape-casted and processed into a capacitor structure, for use in an application such as a magnetic field sensor (see Fig. 6). ${ }^{35,36}$ The material could furthermore be textured by templated grain-growth (TGG) or in situ application of magnetic field during sintering. ${ }^{37}$ Texturing is necessary to achieve a net macroscopic ME coupling coefficient for an otherwise isotropic polycrystalline ME composite. In that way, a cheap yet highly sensitive ME sensor might be obtained, that is compatible with existing large-scale processes from multilayer ceramic capacitor production.

\section{Methods}

Samples for scanning probe microscopy were polished using a route as described in the literature. ${ }^{24,26}$ PFM and MFM experiments were carried out on a 5420 AFM by Agilent Technologies (now Keysight) and a Bruker Dimension Icon, respectively. For PFM experiments, DCP11 conductive diamond coated tips by NT-MDT were used. Diamond coated tips were found to give better signal stability as metal coated tips. ${ }^{26}$ PFM was carried out in the low AC-frequency regime far away from the contact resonance $(70 \mathrm{kHz})$. PPP-MFMR AFM tips by nanosensors were used for MFM experiments in a constant frequency mode. For 


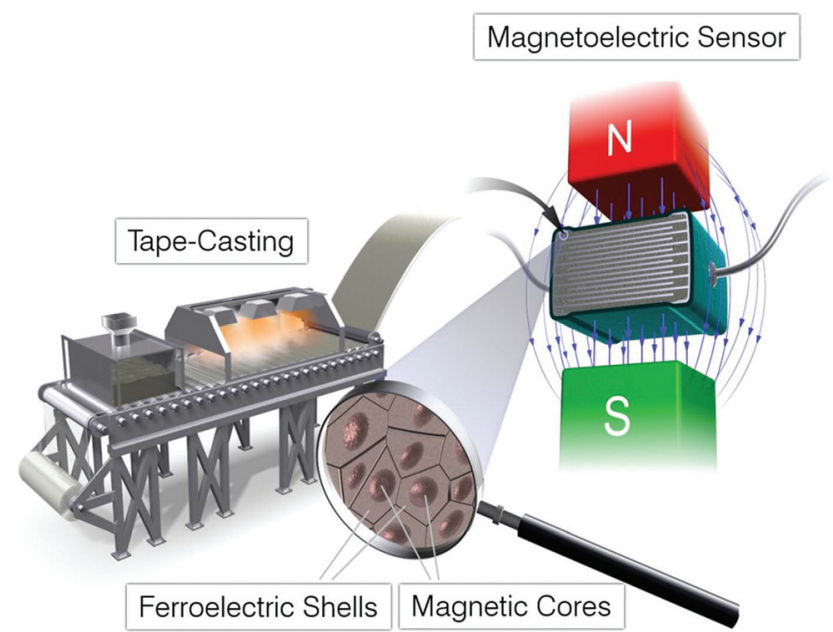

Fig. 6 Outlook: potential application of magnetoelectric core-shell ceramics. 3D-Illustration of tape-casting process (left) for preparation of magnetoelectric core-shell ceramics that could be used in a magnetoelectric magnetic field sensor based on a conventional multilayer ceramic capacitor design (right). The fact that magnetoelectric coreshell ceramics show strong magnetoelectric coupling and can be produced via conventional solid-state synthesis in a single sintering step makes them appealing for applications. Core-shell ceramics produced by tape-casting are already used in commercial multilayer ceramic capacitors.

finding the same micrometer sized area on one sample with different methods, a polished sample was structured using a simple shadow masking method. ${ }^{38}$ Magnetic fields for in situ under magnetic field PFM experiments were generated by the Magnetic Lateral Field Module 5420 by ScienTec with magnetic fields of up to \pm 750 Oe.

AFM and SEM investigations were carried out on three different samples on at least 20 different sample regions. Therefore, we are confident to say that the experimental results presented in Fig. 3 and 5 are representative.

Focused-ion-beam milling (FIB) on an FEI Strata 400S was employed for preparation of electron transparent lamellae. Altogether four samples were investigated by TEM, which all showed similar features as presented in Fig. 2. HRTEM measurements were carried out on an aberration (image) corrected FEI Titan 80-300 operated at $300 \mathrm{kV}$. STEM-EDX maps were obtained on a Philips Tecnai F20 operated at $200 \mathrm{kV}$. For quantification of EDX elemental analysis, the TIA software package from FEI company was used and a thickness correction was applied. The thickness of the sample was estimated based the electron inelastic mean free path as determined by electron energy loss spectroscopy (EELS) using a Gatan GIF Tridiem 863 spectrometer.

Electron probe microanalysis (EPMA) was done on a Jeol JXA 8530-F equipped with a field emission gun operated at 15 $\mathrm{kV}$ with $20 \mathrm{nA}$ probe current. For quantitative elemental analysis, standards were used for each element. For Fe, Co, Ti: pure elemental standards; O: $\mathrm{ZrO}_{2} ; \mathrm{Bi}: \mathrm{Bi}_{2} \mathrm{Se}_{3} ; \mathrm{K}$ : Orthoclase $\left(\mathrm{KAlSi}_{3} \mathrm{O}_{8}\right)$.
For neutron diffraction experiments, the two-axis diffractometer D20 at ILL (Grenoble, France) was used. ${ }^{33}$ The wavelength of neutrons was $2.41 \AA$ using an HOPG (002) monochromator in reflection, at $42^{\circ}$ take-off angle, while a position sensitive detector (PSD), covering a continuous $2 \theta$ range of $153.6^{\circ}$ over a total solid angle of $0.27 \mathrm{sr}$, was used. Five ceramic pellets, from the same material batch and a total weight of approx. $20 \mathrm{~g}$, were stacked into a vanadium can to achieve a high signal intensity.

Scanning-transmission X-ray microscopy was carried out on the PolLux beamline of the Swiss light source (SLS) at the Paul Scherrer Insitut in Switzerland. ${ }^{39-41}$ The X-ray beam was monochromatized to a photon energy resolution of $1.6 \mathrm{eV}$, and focused by a zone plate with an outermost zone width of $30 \mathrm{~nm}$, resulting in a spatial resolution of approx. $45 \mathrm{~nm}$. Each spectrum was normalized by a background spectrum that was recorded at the same time as the sample spectrum to remove instabilities of the X-ray beam intensity.

\section{Abbreviations}

$\begin{array}{ll}\text { ME } & \text { Magnetoelectric coupling } \\ \text { PFM } & \text { Piezoresponse force-microscopy } \\ \text { MFM } & \text { Magnetic force-microscopy } \\ \text { ISCS } & \text { In situ core-shell } \\ \text { TEM } & \text { Transmission electron-microscopy } \\ \text { SEM } & \text { Scanning electron-microscopy } \\ \text { EDX } & \text { Energy dispersive X-ray spectroscopy } \\ \text { EPMA } & \text { Electron-probe microanalysis } \\ \text { HAADF } & \text { High-angle annular dark-field } \\ \text { FFT } & \text { Fast-Fourier-Transform } \\ \text { CFO } & \text { Cobalt ferrite } \\ \text { BFO } & \text { Bismuth ferrite } \\ \text { BKT } & \text { Bismuth potassium titanate } \\ \text { BFC-BKT } & \left.\text { (BiFe }_{0.9} \text { Co }_{0.1} \mathrm{O}_{3}\right)_{0.4}-\left(B_{1 / 2} \mathrm{~K}_{1 / 2} \text { TiO }_{3}\right)_{0.6} \\ \text { CIGM } & \text { Chemically induced grain-boundary migration } \\ \text { EELS } & \text { Electron energy loss spectroscopy } \\ \text { STXM } & \text { Scanning-transmission X-ray microscopy } \\ \text { XAS } & \text { X-ray absorption spectroscopy } \\ \text { E-CFO } & \text { Epitaxial CFO } \\ \text { E-Per } & \text { Epitaxial perovskite } \\ \text { NE-CFO } & \text { Non-epitaxial CFO } \\ \text { NE-Per } & \text { Non-epitaxial perovskite } \\ & \end{array}$

\section{Author contributions}

The manuscript was written through contributions of all authors. All authors have given approval to the final version of the manuscript.

\section{Conflicts of interest}

There are no conflicts to declare. 


\section{Acknowledgements}

Part of the experiments were made possible by Karlsruhe Nano Micro Facility (KNMF, proposals 2016-017-015290 and 2016016-014529). Supporting experiments were performed at the soft X-ray analytics facility WERA at the KIT synchrotron KARA. We are grateful to the KIT synchrotron KARA and to the KNMF (both Karlsruhe, Germany), to the synchrotron SLS (proposal 20171363) of the PSI and to the neutron source ILL (proposal 5-31-2495) for the provision of beamtime. The PolLux end station was financed by the German Ministerium für Bildung und Forschung (BMBF) through contracts 05KS4WE1/6 and 05KS7WE1. LFH wishes to express his deep gratitude to Prof. Frank Schilling for many fruitful discussions and his support.

\section{References}

1 R. Ghosh Chaudhuri and S. Paria, Core/Shell Nanoparticles: Classes, Properties, Synthesis Mechanisms, Characterization, and Applications, Chem. Rev., 2012, 112(4), 2373-2433, DOI: 10.1021/cr100449n.

2 H. Saito, H. Chazono, H. Kishi and N. Yamaoka, X7R Multilayer Ceramic Capacitors with Nickel Electrodes, Jpn. J. Appl. Phys., 1991, 30(Part 1, No. 9B), 2307-2310. Available from: http://stacks.iop.org/1347-4065/30/2307.

3 D. Hennings and G. Rosenstein, Temperature-Stable Dielectrics Based on Chemically Inhomogeneous BaTiO3, J. Am. Ceram. Soc., 1987, 67(4), 249-254, DOI: 10.1111/ j.1151-2916.1984.tb18841.x.

4 M. Acosta, L. A. Schmitt, L. Molina-Luna, M. C. Scherrer, M. Brilz, K. G. Webber, et al., Core-Shell Lead-Free Piezoelectric Ceramics: Current Status and Advanced Characterization of the $\mathrm{Bi}_{1 / 2} \mathrm{Na}_{1 / 2} \mathrm{TiO}_{3}-\mathrm{SrTiO}_{3}$ System. Johnson D, editor, J. Am. Ceram. Soc., 2015, 98(11), 34053422, DOI: $10.1111 /$ jace.13853.

5 T.-H. Song and C. A. Randall, Copper Cofire X7R Dielectrics and Multilayer Capacitors Based on Zinc Borate Fluxed Barium Titanate Ceramic, J. Electroceramics, 2003, 10(1), 39-46. Available from: http://link.springer.com/10.1023/ A:1024028024779.

6 C. A. Randall, S. F. Wang, D. Laubscher, J. P. Dougherty and W. Huebner, Structure property relationships in coreshell $\mathrm{BaTiO}_{3}{ }^{-}$LiF ceramics, J. Mater. Res., 1993, 8(4), 871879. Available from: https://www.cambridge.org/core/article/ structure-property-relationships-in-coreshell-batio3lif-ceramics/ 79583DBD99026A08699C202600020C46.

7 W. Jo, R. Dittmer, M. Acosta, J. Zang, C. Groh, E. Sapper, et al., Giant electric-field-induced strains in lead-free ceramics for actuator applications - status and perspective, J. Electroceramics, 2012, 29(1), 71-93. Available from: http:// link.springer.com/10.1007/s10832-012-9742-3.

8 M. Acosta, L. A. Schmitt, C. Cazorla, A. Studer, A. Zintler, J. Glaum, et al., Piezoelectricity and rotostriction through polar and non-polar coupled instabilities in bismuth-based piezoceramics, Sci. Rep., 2016, 6, 28742, DOI: 10.1038/ srep28742.

9 M. T. Buscaglia, M. Viviani, Z. Zhao, V. Buscaglia and P. Nanni. Synthesis of BaTiO3 Core-Shell Particles and Fabrication of Dielectric Ceramics with Local Graded Structure, 2006, DOI: 10.1021/cm060403j.

10 M. Etier, Y. Gao, V. V. Shvartsman, A. Elsukova, J. Landers, H. Wende, et al., Cobalt Ferrite/Barium Titanate Core/Shell Nanoparticles, Ferroelectrics, 2012, 438(1), 115-122, DOI: 10.1080/00150193.2012.743773.

11 J. Wang, T. Tsuzuki, L. Sun and X. Wang, Reverse Microemulsion-Mediated Synthesis of $\mathrm{SiO}_{2}$-Coated $\mathrm{ZnO}$ Composite Nanoparticles: Multiple Cores with Tunable Shell Thickness, ACS Appl. Mater. Interfaces, 2010, 2(4), 957-960, DOI: 10.1021/am100051z.

12 J. Adam and D. Pendlebury, Global Research Report: Materials Science \& Technology, 2013.

13 D. M. Evans, A. Schilling, A. Kumar, D. Sanchez, N. Ortega, M. Arredondo, et al., Magnetic switching of ferroelectric domains at room temperature in multiferroic PZTFT, Nat. Commun., 2013, 4, 1534, DOI: 10.1038/ncomms2548.

14 L. Keeney, T. Maity, M. Schmidt, A. Amann, N. Deepak, N. Petkov, et al., Magnetic Field-Induced Ferroelectric Switching in Multiferroic Aurivillius Phase Thin Films at Room Temperature, J. Am. Ceram. Soc., 2013, 96(8), 23392357, DOI: 10.1111/jace.12467.

15 H. Zheng, J. Wang, S. E. Lofland, Z. Ma, L. MohaddesArdabili, T. Zhao, et al., Multiferroic $\mathrm{BaTiO}_{3}-\mathrm{CoFe}_{2} \mathrm{O}_{4}$ Nanostructures, Science, 2004, 303(5658), 661-663.

16 G. Schileo, Recent developments in ceramic multiferroic composites based on core/shell and other heterostructures obtained by sol-gel routes, Prog. Solid State Chem., 2013, 41(4), 87-98. Available from: http://linkinghub.elsevier. com/retrieve/pii/S0079678613000186.

17 M. Etier, V. V. Shvartsman, S. Salamon, Y. Gao, H. Wende and D. C. Lupascu, The Direct and the Converse Magnetoelectric Effect in Multiferroic Cobalt FerriteBarium Titanate Ceramic Composites. Raveau B, editor, J. Am. Ceram. Soc., 2016, 99(11), 3623-3631, DOI: 10.1111/ jace.14362.

18 C.-W. Nan, M. I. Bichurin, S. Dong, D. Viehland and G. Srinivasan, Multiferroic magnetoelectric composites: Historical perspective, status, and future directions, J. Appl. Phys., 2008, 103(3), 31101, DOI: 10.1063/1.2836410.

19 L. Yan, Z. Wang, Z. Xing, J. Li and D. Viehland, Magnetoelectric and multiferroic properties of variously oriented epitaxial $\mathrm{BiFeO} 3-\mathrm{CoFe} 2 \mathrm{O} 4$ nanostructured thin films, J. Appl. Phys., 2010, 107(6), 064106, DOI: 10.1063/ 1.3359650 .

20 G. Sreenivasulu, M. Popov, R. Zhang, K. Sharma, C. Janes, A. Mukundan, et al., Magnetic field assisted self-assembly of ferrite-ferroelectric core-shell nanofibers and studies on magneto-electric interactions, Appl. Phys. Lett., 2014, 104(5), 052910, DOI: 10.1063/1.4864113.

21 Q. Zhu, Y. Xie, J. Zhang, Y. Liu, Q. Zhan, H. Miao, et al., Multiferroic $\mathrm{CoFe} 2 \mathrm{O} 4-\mathrm{BiFeO} 3$ core-shell nanofibers and 
their nanoscale magnetoelectric coupling, J. Mater. Res., 2014, 29(05), 657-664. Available from: http://www.journals. cambridge.org/abstract_S0884291414000363.

22 H. Palneedi, V. Annapureddy, S. Priya and J. Ryu, Status and Perspectives of Multiferroic Magnetoelectric Composite Materials and Applications, Actuators, 2016, 5(4), 9. Available from: http://www.mdpi.com/2076-0825/5/ $1 / 9$.

23 R. Adnan Islam and S. Priya, Progress in Dual (Piezoelectric-Magnetostrictive) Phase Magnetoelectric Sintered Composites, Adv. Condens. Matter Phys., 2012, 2012, 1-29. Available from: http://www.hindawi.com/journals/acmp/2012/320612/.

24 L. F. Henrichs, O. Cespedes, J. Bennett, J. Landers, S. Salamon, C. Heuser, et al., Multiferroic Clusters: A New Perspective for Relaxor-Type Room-Temperature Multiferroics, Adv. Funct. Mater., 2016, 26(13), 2111-2121, DOI: $10.1002 /$ adfm.201503335.

25 J. T. Bennett, Development of bismuth ferrite derived piezoelectric ceramics for high temperature applications, University of Leeds, 2014.

26 L. F. Henrichs, J. Bennett and A. J. Bell, Choice of tip, signal stability, and practical aspects of piezoresponseforce-microscopy, Rev. Sci. Instrum., 2015, 86(8), 083707.

27 E. Soergel, Piezoresponse force microscopy (PFM), J. Phys. D: Appl. Phys., 2011, 44(46), 464003. Available from: http:// stacks.iop.org/0022-3727/44/i=46/a=464003.

28 T. Yamada, N. Niizeki and H. Toyoda, Piezoelectric and Elastic Properties of Lithium Niobate Single Crystals, Jpn. J. Appl. Phys., 1967, 6(2), 151. Available from: http:// stacks.iop.org/1347-4065/6/i=2/a=151.

29 H. Matsuo, Y. Noguchi, M. Miyayama, M. Suzuki, A. Watanabe, S. Sasabe, et al., Structural and piezoelectric properties of high-density (Bi0.5K0.5)TiO3 -BiFeO3 ceramics, J. Appl. Phys., 2010, 108(10), 104103. Available from: http://scitation.aip.org/content/aip/journal/jap/108/10/ 10.1063/1.3506717.

30 M. I. Morozov, M.-A. Einarsrud and T. Grande, Polarization and strain response in (Bi0.5K0.5) TiO3 - $\mathrm{BiFeO} 3$ ceramics, Appl. Phys. Lett., 2012, 101(25), 252904, Available from: http://scitation.aip.org/content/aip/journal/apl/101/25/10.1063/ 1.4772588 .

31 D. I. Woodward and I. M. Reaney, Electron diffraction of tilted perovskites, Acta Crystallogr., Sect. B: Struct. Sci., 2005, 61(4), 387-399. Available from: http://scripts.iucr.org/ cgi-bin/paper?S0108768105015521.
32 T. Ozaki, H. Matsuo, Y. Noguchi, M. Miyayama and S. Mori, Microstructures Related to Ferroelectric Properties in (Bi0.5K0.5)TiO3 -BiFeO3, Jpn. J. Appl. Phys., 2010, 49(9S), 09MC05. Available from: http://stacks.iop.org/1347-4065/49/ $\mathrm{i}=9 \mathrm{~S} / \mathrm{a}=09 \mathrm{MC05}$.

33 R. Kiyanagi, T. Yamazaki, Y. Sakamoto, H. Kimura, Y. Noda, K. Ohyama, et al., Structural and Magnetic Phase Determination of (1-x)BiFeO3-xBaTiO3 Solid Solution, J. Phys. Soc. Jpn., 2012, 81(2), 24603, DOI: 10.1143/ JPSJ.81.024603.

34 E. V. Ramana, A. Mahajan, M. P. F. Graça, A. Srinivas and M. A. Valente, Ferroelectric and magnetic properties of magnetoelectric $\left(\mathrm{Na}_{0.5} \mathrm{Bi}_{0.5}\right) \mathrm{TiO}_{3}-\mathrm{BiFeO}_{3}$ synthesized by acetic acid assisted sol-gel method, J. Eur. Ceram. Soc., 2014, 34(16), 4201-4211. Available from: http://www.sciencedirect.com/science/article/pii/S0955221914003550.

35 C. Israel, N. D. Mathur and J. F. Scott, A one-cent roomtemperature magnetoelectric sensor, Nat. Mater., 2008, 7(2), 93-94, DOI: 10.1038/nmat2106.

36 C. Israel, S. Kar-Narayan and N. D. Mathur, Eliminating the Temperature Dependence of the Response of Magnetoelectric Magnetic-Field Sensors, IEEE Sens. J., 2010, 10(5), 914-917. Available from: http://ieeexplore.ieee. org/document/5437517/.

37 W. Bai, B. Shen, F. Fu and J. Zhai, Fabrication and Electrical Properties of $\mathrm{Ba}(\mathrm{Zr}, \mathrm{Ti}) \mathrm{O}$ \$_ $\{3\}$ \$ Textured Ceramics by Templated Grain Growth, Jpn. J. Appl. Phys., 2012, 51(1), 015503. Available from: http://stacks.iop.org/1347-4065/51/ 015503.

38 L. F. Henrichs, L. Chen and A. J. Bell, Simple technique for high-throughput marking of distinguishable micro-areas for microscopy, J. Microsc., 2016, 262(1), 28-32.

39 U. Flechsig, C. Quitmann, J. Raabe, M. Böge, R. Fink and H. Ade, The PolLux Microspectroscopy Beam line at the Swiss Light Source, in AIP Conference Proceedings, AIP, 2007, pp. 505-508. DOI: 10.1063/1.2436109.

40 J. Raabe, G. Tzvetkov, U. Flechsig, M. Böge, A. Jaggi, B. Sarafimov, et al., PolLux: A new facility for soft X-ray spectromicroscopy at the Swiss Light Source, Rev. Sci. Instrum., 2008, 79(11), 113704, DOI: 10.1063/1.3021472.

41 U. Frommherz, J. Raabe, B. Watts, R. Stefani, U. Ellenberger and R. Garrett, et al., Higher Order Suppressor (HOS) for the PolLux Microspectroscope Beamline at the Swiss Light Source SLS, in AIP Conference Proceedings, American Institute of Physics, 2010, pp. 429-432. DOI: 10.1063/1.3463232. 